\title{
Realization of the Noncommutative Seiberg-Witten Gauge Theory by Fields in Phase Space
}

\author{
R.G.G. Amorim, ${ }^{1,2}$ F.C. Khanna, ${ }^{3,4}$ A.P.C. Malbouisson, ${ }^{5}$ J.M.C. Malbouisson, ${ }^{6}$ and A.E. Santana ${ }^{2}$ \\ ${ }^{1}$ Faculdade Gama,Universidade de Brasilia, 72444-240, Brasília, DF, Brazil \\ ${ }^{2}$ International Centre for Condensed Matter Physics Instituto de Física, \\ Universidade de Brasília, 70910-900, Brasília, DF, Brazil \\ ${ }^{3}$ Department of Physics and Astronomy, University of Victoria, 3800 Finnerty Road Victoria,BC V8P 5C2 \\ ${ }^{4}$ TRIUMF, 4004, Westbrook mall, Vancouver, British Columbia V6T 2A3, Canada \\ ${ }^{5}$ Centro Brasileiro de Pesquisas Físicas, Rua Dr. X. Sigaud 150, 22290-180, Rio de Janeiro, RJ, Brazil \\ ${ }^{6}$ Instituto de Física, Universidade Federal da Bahia, 40210-340, Salvador, BA, Brazil
}

\begin{abstract}
Representations of the Poincaré symmetry are studied by using a Hilbert space with a phase space content. The states are described by wave functions (quasi amplitudes of probability) associated with Wigner functions (quasi probability density). The gauge symmetry analysis provides a realization of the Seiberg-Witten gauge theory for noncommutative fields.
\end{abstract}

PACS numbers:

\section{INTRODUCTION}

Considering the association of noncommutative geometry with string theory, Seiberg and Witten[1] studied a string dynamics described by a minimally coupled supersymmetric gauge field in a non-commutative space. The result is a generalized gauge field $A^{\mu}$ with an antisymmetric tensor field given by $F^{\mu \nu}=\partial_{\nu} A^{\mu}-$ $\partial_{\mu} A^{\nu}-i\left\{A^{\mu}, A^{\nu}\right\}_{M}$, where $\left\{A^{\mu}, A^{\nu}\right\}_{M}$ is the MoyalPoisson bracket. Here we present a realization of such a noncommutative gauge theory, starting with representations of space-time symmetries in a phase space manifold. In particular, we show that correlation functions are closely associated with the Wigner function, describing bosons and fermions.

The idea of noncommutativity in $\mathbb{R}^{3}$ starts with a suggestion by Heisenberg [3], which was realized by Snyder [4, 5] and Yang [6], by exploring the de Sitter space. The algebraic structure of non-commutative geometries has been developed along different lines, in particular in association with $\mathrm{c}^{*}$-algebras [7]. The interest in noncommutative theories, since the $1990 \mathrm{~s}$, is due to a variety of applications [1, 8], including abelian and non-abelian gauge theories 8 11], gravity [12, 13], standard model for particles [15, 16], supersymmetry [17] and in quantum Hall effect 18]. Issues such as the ultraviolet/infrared mixing and the renormalizability of noncommutative theories have been also addressed [19 21].

The compatibility of non-commutative geometries and the Lorentz space-time symmetry has been achieved with the twisted noncommutative theories [22 24]. However, this type of compatibility arises also in the Wignerfunction formalism [25, 26], initially proposed for developments in quantum kinetic theory. The algebraic structures and applications of Wigner-function are of interest in a vast range of areas, including noncommutative geometries and relativistic theories [26-40]. It is important to mention the use of this method in experiments considering the reconstruction of quantum states and measure- ments of Wigner function in quantum tomography 41 45.

Associated with the algebraic structure of the Wigner formalism, there are studies exploring the notion of wave function in phase-space [32, 33, 36, 37], a crucial step to develop abelian and non-abelian gauge theories, with a close connection to Wigner theory. However, several aspects in these methods remain to be clarified; the most important being a rigorous association of wave functions in phase-space (or the quasi-amplitude of probability) with the Wigner function (the quasi distribution of probability), in order to provide a physical interpretation for the formalism [32, 33, 46 48]. This problem is addressed here in order to carry out an analysis of a gauge theory associated with the Wigner function. This leads to a realization of the Seiberg-Witten gauge theory for noncommutative fields. We start, in Section 2, by presenting the Poincaré-Lie algebra, using a carrier Hilbert space with the content of phase space. This is used in Section 3 to derive spin zero and spin 1/2 representations. Then, in Section 4, the gauge theory is constructed for bosons and fermions. Some final concluding remarks are presented in Section 5.

\section{PONCARÉ GROUP IN PHASE SPACE}

Let $\mathbb{M}$ be an analytical manifold where each point is specified by Minkowski coordinates $q^{\mu}$, with $\mu=0,1,2,3$, and a metric such that $\operatorname{diag}(g)=(+---)$. Let $T^{*} \mathbb{M}$ be the cotangent-bundle, where each point is specified by the coordinates $\left(q^{\mu}, p^{\mu}\right)$. We use this twofold structure of $T^{*} \mathbb{M}$ to construct a standard representation for the $\mathrm{c}^{*}$-algebra with the content of a phase space.

We take advantage of the fact that $T^{*} \mathbb{M}$ can be equipped with a symplectic structure via a 2 -form $\omega=$ $d q^{\mu} \wedge d p_{\mu}$, called the symplectic form. Let us define the 
operator on $C^{\infty}\left(T^{*} M\right)$,

$$
\Lambda=\frac{\overleftarrow{\partial}}{\partial q^{\mu}} \frac{\vec{\partial}}{\partial p_{\mu}}-\frac{\overleftarrow{\partial}}{\partial p^{\mu}} \frac{\vec{\partial}}{\partial q_{\mu}}
$$

such that for $C^{\infty}$ functions, $f(q, p)$ and $g(q, p)$, we have

$$
\omega(f \Lambda, g \Lambda)=f \Lambda g=\{f, g\}
$$

where

$$
\{f, g\}=\frac{\partial f}{\partial q^{\mu}} \frac{\partial g}{\partial p_{\mu}}-\frac{\partial f}{\partial p^{\mu}} \frac{\partial g}{\partial q_{\mu}}
$$

is the Poisson bracket. The space $T^{*} \mathbb{M}$ endowed with this symplectic structure is called the phase space, and will be denoted by $\Gamma$.

In order to construct a Hilbert space in $C^{\infty}(\Gamma)$, let $\mathcal{H}(\Gamma)$ be a linear subspace of the space of measurable functions $\psi: \Gamma \rightarrow \mathbb{C}$ which are square integrable, i.e. such that

$$
\int_{\Gamma} d^{4} p d^{4} q \psi^{*}(q, p) \psi(q, p)<\infty
$$

The Hilbert space is introduced by defining the inner product, $\langle\cdot \mid \cdot\rangle$, on $\mathcal{H}(\Gamma)$, as

$$
\left\langle\psi_{1} \mid \psi_{2}\right\rangle=\int_{\Gamma} \psi_{1}(q, p)^{*} \psi_{2}(q, p) d^{4} p d^{4} q
$$

where we take $\left(q^{\mu}, p^{\mu}\right)=(q, p)$, and $\psi(q, p)$ in $C^{\infty}(\Gamma)$. In this case we have $\psi(q, p)=\langle q, p \mid \psi\rangle$, with

$\int d^{4} p d^{4} q|q, p\rangle\langle q, p|=1$ and $\left\langle q, p \mid q^{\prime}, p^{\prime}\right\rangle=\delta\left(q-q^{\prime}\right) \delta\left(p-p^{\prime}\right)$.

Using the kets $|q, p\rangle$, we define the following c-number operators $\bar{Q}$ and $\bar{P}$ by

$$
\bar{Q}|q, p\rangle=q|q, p\rangle, \quad \bar{P}|q, p\rangle=p|q, p\rangle,
$$

fulfilling the commutation condition $[\bar{Q}, \bar{P}]=0$. This Hilbert space is taken here as the representation space of the Poincaré symmetries. For the sake of physical interpretation, the state of a system will be described by functions $\psi(q, p)$, with the normalization condition

$$
\langle\psi \mid \psi\rangle=\int d^{4} p d^{4} q \psi^{*}(q, p) \psi(q, p)=1
$$

A unitary transformation in $\mathcal{H}(\Gamma)$ is the mapping $U$ : $\mathcal{H}(\Gamma) \rightarrow \mathcal{H}(\Gamma)$ such that $\left\langle\psi_{1} \mid \psi_{2}\right\rangle$ is invariant. We consider the mapping $U_{\Delta}=\exp (-i \hbar \Delta / 2)$, where $\Delta=\frac{\partial}{\partial q^{\mu}} \frac{\partial}{\partial p_{\mu}}$. This linear transformation gives rise to the following basic operators

$$
\begin{aligned}
& P^{\mu}=U_{\Delta} \bar{P}^{\mu} U_{\Delta}^{-1}=p^{\mu}-\frac{i \hbar}{2} \frac{\partial}{\partial q_{\mu}} \\
& Q^{\mu}=U_{\Delta}^{-1} \bar{Q}^{\mu} U_{\Delta}=q^{\mu}+\frac{i \hbar}{2} \frac{\partial}{\partial p_{\mu}} .
\end{aligned}
$$

In natural units $(\hbar=c=1)$, which, unless explicitly stated, we use from now on, these operators satisfy the following commutation relation:

$$
\left[Q^{\mu}, P^{\nu}\right]=i g^{\mu \nu}
$$

Then we construct a representation for the Poincaré group in phase space, by defining

$$
M_{\mu \nu}=Q^{\mu} P^{\nu}-P^{\nu} Q^{\mu}
$$

such that the Poincaré Lie algebra is given by

$$
\begin{aligned}
{\left[M_{\mu \nu}, P_{\sigma}\right] } & =i\left(g_{\nu \sigma} P_{\mu}-g_{\sigma \mu} P_{\nu}\right), \\
{\left[M_{\sigma \rho}, M_{\mu \nu}\right] } & =i\left(g_{\mu \rho} M_{\nu \sigma}-g_{\nu \rho} M_{\mu \sigma}\right. \\
& \left.+g_{\mu \sigma} M_{\rho \nu}-g_{\nu \sigma} M_{\rho \mu}\right)
\end{aligned}
$$

other commutation relations are zero. Explicitly, we have

$$
\begin{aligned}
M^{\mu \nu} & =q^{\mu} p^{\nu}-q^{\nu} p^{\mu}-\frac{i}{2} q^{\mu} \frac{\partial}{\partial q_{\nu}}+\frac{i}{2} q^{\nu} \frac{\partial}{\partial q_{\mu}} \\
& -\frac{i}{2} p^{\mu} \frac{\partial}{\partial p_{\nu}}+\frac{i}{2} p^{\nu} \frac{\partial}{\partial p_{\mu}} \\
& +\frac{i}{4} \frac{\partial^{2}}{\partial p_{\mu} \partial q_{\nu}}-\frac{i}{4} \frac{\partial^{2}}{\partial p_{\nu} \partial q_{\mu}} .
\end{aligned}
$$

In order to construct representations, we use the Casimir invariants

$$
P^{2}=m^{2} \text { and } w_{\mu} w^{\mu}=-m^{2} s(s+1),
$$

where

$$
w_{\mu}=\frac{1}{2} \varepsilon_{\mu \nu \rho \sigma} M^{\nu \sigma} P^{\rho}=-m^{2} s(s+1)
$$

is the Pauli-Lubanski vector.

\section{SCALAR AND DIRAC FIELDS IN PHASE SPACE}

For the scalar representation, we have $s=0$, such that $P^{\mu} P_{\mu} \phi(p, q)=m^{2} \phi(p, q)$, that leads to

$$
\frac{-1}{4} \frac{\partial^{2} \phi(p, q)}{\partial q^{\mu} \partial q_{\mu}}-i p^{\mu} \frac{\partial \phi(p, q)}{\partial q^{\mu}}+\left(p^{\mu} p_{\mu}-m^{2}\right) \phi(p, q)=0 .
$$

This is a Klein-Gordon-like equation written in phase space. In order to find the physical meaning for such an equation, we show the association of $\phi(p, q)$ with the Wigner function. Indeed it has been shown that [46, 47]

$$
f_{W}(q, p)=\phi(q, p) \star \phi^{\dagger}(q, p),
$$

satisfies the equation

$$
\left\{p^{2}, f_{W}(q, p)\right\}_{M}=p_{\mu} \frac{\partial}{\partial q^{\mu}} f_{W}(q, p)=0,
$$


such that

$$
\{f(q, p), g(q, p)\}_{M}=f(q, p) \star g(q, p)-g(q, p) \star f(q, p)
$$

is the Moyal bracket, where the star product given by

$$
A_{W}(q, p) e^{\frac{i \Lambda}{2}} B_{W}(q, p)=A_{W}(q, p) \star B_{W}(q, p) .
$$

Thus we introduce the Wigner mapping in the following way. Let $\widehat{A}$ be an operator acting in the Hilbert space $\mathcal{H}$. The Wigner mapping $W: \widehat{A} \rightarrow A_{W}(q, p)$ is defined by

$$
A_{W}(q, p)=\frac{1}{\sqrt{2 \pi}} \int d z \exp (i p z)\left\langle q-\frac{z}{2}|\widehat{A}| q+\frac{z}{2}\right\rangle .
$$

Considering such a mapping for $f_{W}(q, p)$, with $W: \rho \rightarrow$ $f_{W}(q, p)$, then we show that [29]

$$
\left[\frac{\partial^{2}}{\partial q^{\mu} \partial q_{\mu}}, \rho\right]=0
$$

This is a Liouville-von Neumann-like equation. Since $\int d q d p f_{W}(q, p)=1$, we have $\operatorname{Tr} \rho=1$, implying that $\rho$ is a density matrix and $f_{W}(q, p)$ is a Wigner function derived from $\rho$. In addition, it is important to observe that if we consider, for instance, $\rho(q)=\phi(q) \phi^{\dagger}(q)$, with $\phi(q) \in \mathcal{H}$, then $\phi(q)$ satisfies the Klein-Gordon equation,

$$
\left(\frac{\partial^{2}}{\partial q^{\mu} \partial q_{\mu}}-m^{2}\right) \phi(q)=0 .
$$

An operator $\widehat{A}$ acting on the Hilbert space $\mathcal{H}$ is mapped into a Wigner representation by $W: A \rightarrow A_{W}(q, p)$, which in turn is mapped in operators acting on $\mathcal{H}(\Gamma)$, such as

$$
\Omega: A_{W}(q, p) \rightarrow A(Q, P)=A_{W}(q, p) \star .
$$

Then we derive the composed mapping

$$
\Omega \circ W: \widehat{A} \rightarrow A(Q, P) .
$$

As an example, consider, respectively, the position and momentum operators, $\widehat{q}$ and $\widehat{p}$ defined by acting on the Hilbert space $\mathcal{H}$, we have

$$
(\Omega \circ W)\left(\widehat{q}^{\mu}\right)=Q^{\mu}=q^{\mu} \star, \quad(\Omega \circ W)\left(\widehat{p}^{\mu}\right)=P^{\mu}=p^{\mu} \star
$$

where the operators $Q^{\mu}$ and $P^{\mu}$ were first introduced in Eq. (5) and Eq. (6), respectively. In addition, we prove the following identity for the average of an observable $A(P, Q)$ in a state $|\phi\rangle \in \mathcal{H}(\Gamma)$ :

$$
\begin{aligned}
\langle A\rangle & =\langle\phi|A(P, Q)| \phi\rangle \\
& =\int d q d p \phi^{\dagger}(q, p) A_{W}(q, p) \star \phi(q, p) \\
& =\int d q d p A(q, p) f_{W}(q, p)=\operatorname{Tr} \rho A,
\end{aligned}
$$

where we have used

$$
A(P, Q)=A_{W}(q, p) \star=A_{W}(q \star, p \star) .
$$

It is important to emphasize that the association of $\phi(q, p)$ with the Wigner function provides the physical interpretation of the formalism based on representations in the Hilbert space $\mathcal{H}(\Gamma)$. In this case, since the Wigner function is a quasi-distribution of probability, it is natural to denominate $\phi(q, p)$ as a quasi-amplitude of probability.

Representations for fermions are carried out following standard procedures. Taking $s=1 / 2$ in the PauliLubanski vector, it leads to a Dirac equation in phase space given by [4]

$$
\gamma^{\mu}\left(p_{\mu}-\frac{i}{2} \frac{\partial}{\partial q^{\mu}}\right) \psi=m \psi
$$

where the $\gamma$-matrices satisfy $\left(\gamma^{\mu} \gamma^{\nu}+\gamma^{\nu} \gamma^{\mu}\right)=2 g^{\mu \nu}$. The Lagrangian density is

$$
\mathcal{L}=\frac{-i}{4}\left[\left(\partial_{\mu} \psi\right) \gamma^{\mu} \psi-\bar{\psi}\left(\gamma^{\mu} \partial_{\mu} \psi\right)\right]-\bar{\psi}(q, p)\left(m-\gamma^{\mu} p_{\mu}\right) \psi(q, p)
$$

In a similar manner as for the case of bosons, considered before, the Wigner function associated with the Dirac field in phase space is

$$
f_{W}(q, p)=\psi(q, p) \star \bar{\psi}(q, p),
$$

satisfying the equation of motion

$$
p^{\mu} \frac{\partial f_{W}(q, p)}{\partial q^{\mu}}=0
$$

such that, considering a mapping $W: \rho \rightarrow f_{W}(q, p)$, a Liouville--von Neumann equation is derived:

$$
\left[\gamma^{\mu} \partial_{\mu}, \rho\right]=0
$$

It is worth mentioning that, due to the nature of the probability quasi-amplitude, Eq. (10) and Eq. (13) provide phase-invariant field formalisms in phase space (which is not the case of the usual Wigner function method). This aspect is developed in the following.

\section{SEIBERG-WITTEN GAUGE FIELDS}

The Lagrangian of the free Klein-Gordon field describing $N$-bosons is written as

$$
\mathcal{L}_{0}=\left(p^{\mu} \star \phi\right)\left(p_{\mu} \star \phi^{\dagger}\right)+m^{2} \phi \phi^{\dagger},
$$

where $\phi \equiv \phi(q, p)$. From this lagrangian, Eq. (10) can be derived. Our aim is to analyze the invariance of Eq. (15) under local gauge transformations. In this sense, we take the transformation rules given by

$$
\phi \rightarrow e^{-i \Lambda} \star \phi, \quad \phi^{\dagger} \rightarrow e^{i \Lambda} \star \phi^{\dagger},
$$

and where $\Lambda \equiv \Lambda(q, p)$. For infinitesimal transformation,we have $\delta \phi=-i \Lambda \star \phi$ and $\delta \phi^{\dagger}=i \Lambda \star \phi^{\dagger}$, such that

$$
\delta\left(p^{\mu} \star \phi\right)=-i p^{\mu} \star \Lambda \star \phi,
$$


and

$$
\delta\left(p_{\mu} \star \phi^{\dagger}\right)=i p_{\mu} \star \Lambda \star \phi^{\dagger} .
$$

It should be noted that, $\left(p^{\mu} \star \phi\right)$ and $\left(p_{\mu} \star \phi^{\dagger}\right)$ do not transform covariantly, i.e. not in the same way as $\phi$ and $\phi^{\dagger}$ themselves. To demonstrate this aspect, we define the operator

$$
D^{\mu} \star=p^{\mu} \star-i A^{\mu} \star,
$$

leading to a Lagrangian written as

$$
\mathcal{L}=\left(D^{\mu} \star \phi\right)\left(D_{\mu} \star \phi^{\dagger}\right)+m^{2} \phi \phi^{\dagger} .
$$

Now we have to prove the invariance of this Lagrangian. It follows immediately that

$$
\begin{aligned}
\delta\left(D^{\mu} \star \phi\right) & =-i p^{\mu}(\Lambda \star \phi)-\frac{1}{2} \frac{\partial \Lambda}{\partial q_{\mu}} \star \phi \\
& -\frac{1}{2} \Lambda \star \frac{\partial \phi}{\partial q_{\mu}}-A^{\mu} \star(\Lambda \star \phi)-i\left(\delta A^{\mu}\right) \star \phi
\end{aligned}
$$

Now, using the identity

$$
p(f \star g)=f \star(p g)-\frac{i}{2}\left(\partial_{q} f\right) \star g,
$$

we have

$$
\begin{aligned}
\delta\left(D^{\mu} \star \phi\right) & =-i \Lambda \star\left(p^{\mu} \star \phi\right)-\frac{\partial \Lambda}{\partial q_{\mu}} \star \phi \\
& -A^{\mu} \star(\Lambda \star \phi)-i\left(\delta A^{\mu}\right) \star \phi .
\end{aligned}
$$

Considering a gauge transformations of the second kind, we demand that,

$$
A^{\prime \mu} \rightarrow A^{\mu}+i\left\{A^{\mu}, \Lambda\right\}_{M}+i \frac{\partial \Lambda}{\partial q_{\mu}},
$$

where $\{a, b\}_{M}=a \star b-b \star a$ is the Moyal bracket. Then Eq. (21) reads

$$
\delta\left(D^{\mu} \star \phi\right)=-i \Lambda \star\left(p^{\mu} \star-i A^{\mu} \star\right) \phi ;
$$

i.e.

$$
\delta\left(D^{\mu} \star \phi\right)=-i \Lambda \star\left(D^{\mu} \star \phi\right),
$$

which is the covariant transformation rule.

Similarly, we show that

$$
\delta\left(D_{\mu} \star \phi^{\dagger}\right)=-i \Lambda \star\left(D_{\mu} \star \phi^{\dagger}\right) .
$$

Then we have the following rule for the minimal substitution: replace $p^{\mu} \star$ by $p^{\mu} \star-i A^{\mu} \star$.

The Lagrangian given by Eq. (19) is now invariant. The field $A^{\mu}$, however, must contribute by itself to the Lagrangian. Then we define

$$
F^{\mu \nu}=\frac{\partial A^{\mu}}{\partial q_{\nu}}-\frac{\partial A^{\nu}}{\partial q_{\mu}}-i\left\{A^{\mu}, A^{\nu}\right\}_{M}
$$

so that $F^{\mu \nu}$ is invariant. Then the final Lagrangian is

$$
\mathcal{L}=\left(D^{\mu} \star \phi\right)\left(D_{\mu} \star \phi^{*}\right)+m^{2} \phi \phi^{*}-\frac{1}{4} F^{\mu \nu} F_{\mu \nu} .
$$

This provides a realization of the Seiberg-Witten gauge theory for noncommutative fields.

Proceeding with the usual canonical quantization, we take $\phi$ as an operator acting in a (symplectic) Fock space, such that the two-point function for the free field is defined as

$$
G\left(q-q^{\prime}, p-p^{\prime}\right)=\left\langle 0\left|\mathrm{~T}\left[\phi(q, p) \phi^{*}\left(q^{\prime}, p^{\prime}\right)\right]\right| 0\right\rangle,
$$

where $\mathrm{T}$ is the time-ordering operator. The physical interpretation of $G\left(q-q^{\prime}, p-p^{\prime}\right)$ is obtained by observing that it is related to the Wigner function by [48]

$$
f_{W}(q, p)=\lim _{q^{\prime}, p^{\prime} \rightarrow q, p} \exp i\left(\partial_{q} \partial_{p^{\prime}}-\partial_{q^{\prime}} \partial_{p}\right) G\left(q-q^{\prime}, p-p^{\prime}\right) .
$$

This provides a way to develop a perturbative theory for the Wigner function considering this scalar electrodynamics in phase space. Observe that we have the mapping: $\Omega \circ W: \mathrm{T}\left[\phi(q) \phi^{*}\left(q^{\prime}\right)\right] \rightarrow \mathrm{T}\left[\phi(q, p) \phi^{*}\left(q^{\prime}, p^{\prime}\right)\right]$, such that $\left\langle 0\left|\mathrm{~T}\left[\phi(q) \phi^{*}\left(q^{\prime}\right)\right]\right| 0\right\rangle$ is the usual propagator for the free Klein-Gordon field. For the gauge field, considering the changing $\phi \rightarrow A^{\mu}$, we obtain similar correlation functions, with the same physical interpretation.

Considering then the gauge invariance, we obtain a realization of the Seiberg-Witten gauge theory for fermions

$$
\mathcal{L}=\bar{\psi}(q, p)\left(\gamma^{\mu} D_{\mu} \star \psi(q, p)+m^{2} \bar{\psi}(q, p) \psi(q, p)-\frac{1}{4} F^{\mu \nu} F_{\mu \nu} .\right.
$$

This gauge invariant Lagrangian in phase space provides a theory associated with a Wigner function for spin $1 / 2$ particles.

\section{CONCLUDING REMARKS}

In this paper, we have derived the field theory in phase space, associated with Wigner functions. Taking into account the notion of wave-functions in phase-space (quasiamplitudes of probability), we are able to introduce gauge fields in phase space. Then equations for the quantum electrodynamics in phase space are derived. It is important to emphasize that the gauge symmetry provides a realization of the Seiberg-Witten gauge theory for noncommutative fields. The generalization for non-abelian gauge fields, although intricate, follows along similar lines. This aspect will be analyzed closely elsewhere.

\section{Acknowledgments}

This work was partially supported by CAPES and CNPq of Brazil and NSERC of Canada. 
[1] N. Seiberg, E. Witten, String theory and noncommutative geometry, JHEP, 9909 (1999) 32 hep-th/9908142.

[2] M. R. Douglas, N. A. Nekrasov, Noncommutative field theory. Rev. Mod. Phys. A $\mathbf{7 3}$ (2001) 977 hep-th/0106048.

[3] W. Pauli, Scientific correspondence, Vol II, p.15, Ed. K. von Meyenn (Spring-Verlag, Berlin, 1985).

[4] H. S. Snyder, Quantized space-time, Phys. Rev. 71, 38 (1947).

[5] H. S. Snyder, The Electromagnetic field in quantized space-time, Phys. Rev. 72, 68 (1947).

[6] C. N. Yang, On quantized space-time, Phys. Rev. 72, 874 (1947).

[7] A. Connes, Noncommutative geometry (Academic Press, San Diego, 1990).

[8] R. J. Szabo, Quantum field theory on noncommutative Spaces, Phys. Rep. 378, 207 (2003).

[9] T. Mariz, J. R. Nascimento, V. O. Rivellis, Dispersion relations in noncommutative theories, Phys. Rev. D 75, 025020 (2007).

[10] M. L. Costa, A. R. Queiroz, A. E. Santana, Noncommutative thermofield dynamics, Int. J. Mod. Phys. A 25, 3209 (2010).

[11] R. Gurau, A.P.C. Malbouisson, V. Rivasseau, A.Tanasa, Non-Commutative complete Mellin representation for Feynman amplitudes, Lett. Math. Phys. 81, 161 (2007) [arXiv:math-ph/0705.3437].

[12] W. Kalau, M. Walze, Gravity noncommuative geometry and the Wodzicki residue, J. Geom. Phys. 16, 327 (1955).

[13] D. Kastler, The Dirac operator and gravitation, Commun. Math Phys. 166, 633 (1995).

[14] A. H. Chanseddine, A. Connes, The spectral action principle, Commun. Math. Phys. 186, 731 (1997).

[15] J. C. Varilly, J. M. Gracia-Bondía, Noncommutative differencial geometry and the standard model, J. Geom. Phys. 12, 223 (1993).

[16] C. P Martin, J. C. Varilly, J. M. Gracia-Bondía, The standard model as a noncommutative geometry: the low energy regime, Phys. Rep. 294, 363 (1998).

[17] H. O. Girotti, M. Gomes, V. O. Rivelles, A. J. da Silva, A consistent noncommutative field theory: the Wess-Zumino model, Nucl.Phys. B 587, 299 (2000) hep-th/0005272.

[18] J. Belissard, A. van Elst, H. Schulz-Baldes, The noncommutative geometry of the quantum Hall effect, J. Math. Phys. 35, 53 (1994).

[19] H. Grosse, R. Wilkenhaar, Renormalisation of $\phi^{4}$-theory on noncommutative $\mathbb{R}^{4}$ in the matrix base, Comumm. Math. Phys. 256, 305 (2005).

[20] E. Langmann, R. J. Szabo, Duality in scalar field theory on noncommutative phase spaces, Phys. Lett. B, 533, 168 (2002).

[21] J. Magnen, V. Rivasseau, A. Tanasa, Commutative limit of a renormalizable noncommutative model, EPL $\mathbf{8 6}$, 11001 (2009).

[22] A. P. Balachandran, T. R. Govindarajan, G. Mangano, A. Pinzul, B. A. Qureshi, S. Vaidya, Statistics and $U V$ IR mixing with twisted Poincaré invariance, Phys. Rev. D 75, 045009 (2007).

[23] A. P. Balachandran, A. M. Marques, A. R. Queiroz, P. Teotonio-Sobrinho, Deformed Kac-Moody and Vira- soro algebras, J. Phys. A 40, 7789 (2007).

[24] A. P. Balachandran, P. Padmanabhan, A. R. de Queiroz, Lehmann-Symanzik-Zimmermann S-matrix elements on the Moyal plane, Phys. Rev. D 84, 065020 (2011).

[25] E. P. Wigner, On the quantum correction for thermodynamic equilibrium, Phys. Rev. 40, 749 (1932).

[26] M. Hillery, R. F. O 'Connell, M. O. Scully, E. P. Wigner, Distribution functions in physics: fundamentals, Phys. Rep. 106, 121 (1984).

[27] Y. S. Kim, M. E. Noz, Phase space picture and quantum mechanics - group theoretical approach (W. Scientific, London, 1991).

[28] T. Curtright, D. Fairlie, C. Zachos, Phys. Rev. D 58 25002 (1998).

[29] F. C. Khanna, A. P. C. Malbouisson, J. M. C. Malbouisson, A. E. Santana, Thermal quantum field theory: algebraic aspects and applications (W. Scientific, Singapore, 2009).

[30] H. Weyl, Quantenmechanik und gruppentheorie, Z. Phys. 46, 1 (1927).

[31] J. E. Moyal, Quantum mechanics as a statistical theory, Proc. Camb. Phil. Soc. 45, 99 (1949).

[32] J. Dito, Star-products and nonstandard quantization for Klein-Gordon equation, J. Math. Phys. 33, 791 (1992).

[33] Go. Torres-vega, J.H. Frederick, A quantum mechanical representation in phase space, J. Chem. Phys. 93, 8862 (1990).

[34] O. F. Dayi, L. T. Kelleyane, Wigner function for the Landau problem in noncommutative spaces, Mod. Phys. Lett. A 17, 1937 (2002).

[35] M. A. Alonso, G. S. Pogosyan, K. B. Wolf, Wigner functions for curved spaces. I. On hyperboloids, J. Math. Phys. 43, 5857 (2002).

[36] M. de Gosson, Symplectically covariant Schrödinger equation in phase space, J. Phys. A: Math. Gen. 38, 9263 (2005).

[37] M. de Gosson, F. Luef, On the usefulness of modulation spaces in deformation quantization J. Phys. A: Math. Theor. 42, 315205 (2009).

[38] V. V Dodonov, Wigner functions and statistical moments of quantum states with definite parity, Phys. Lett. A 364, 368 (2007).

[39] V. V. Dodonov, O.V. Man'ko, V. I. Man'ko, Photon distribution for one-mode mixed light with a generic Gaussian Wigner function, Phys. Rev. A 49, 2993 (1994).

[40] M. Berkowitz, Exponential approximation for the density matrix and the Wigner's distribution, Chem. Phys. Lett. 129, 486 (1986).

[41] D. T. Smithey, M. Beck, M. G. Raymer, A. Faridani, Measurement of the Wigner distribution and the density matrix of a light mode using optical homodyne tomography: Application to squeezed states and the vacuum, Phys. Rev. Lett. 70, 1244 (1993).

[42] D. Leibfried, D. M. Meekhof, B. E. King, C. Monroe, W. M. Itano, D. J. Wineland, Experimental Determination of the Motional Quantum State of a Trapped Atom, Phys. Rev. Lett. 77, 4281 (1996).

[43] L. G. Lutterbach, L. Davidovich, Method for Direct Measurement of the Wigner Function in Cavity QED and Ion Traps, Phys. Rev. Lett. 78, 2547 (1997).

[44] R. L. de Matos Filho, W. Vogel, Engineering the Hamil- 
tonian of a trapped atom, Phys. Rev. A 58, R1661 (1998).

[45] A. Ibort, V. I. Man'ko, G. Marmo, A. Simoni, F. Ventriglia, An introduction to the tomographic picture of quantum mechanics, Phys. Scripta 79, 065013 (2009).

[46] M. D. Oliveira, M. C. B. Fernandes, F. C. Khanna, A. E. Santana, J. D. M. Vianna, Sympletic quantum mechanics, Ann. Phys. (N.Y.) 312, 492 (2004).

[47] R. G. G. Amorim, M. C. B. Fernandes, F. C. Khanna, A.
E. Santana, J. D. M. Vianna, Non-commutative geometry and symplectic field theory, Phys. Lett. A 361, 464 (2007).

[48] R. G. G. Amorim, F. C. Khanna, A. E. Santana, J. D. M. Vianna, Perturbative symplectic field theory and Wigner function, Physica A 388, 3771 (2009). 